\title{
Closing the Loop
}

\author{
Revital Nimri ${ }^{1}$, Hena Yakoob ${ }^{2}$, Benjamen Schoenberg ${ }^{2}$, and Eyal Dassau ${ }^{2,3,4}$
}

Intro

Yye 1 Diabetes Mellitus presents a significant challenge to patients, as it involves integrating treatment into daily life to compensate for the inability to produce insulin to meet regular metabolic needs. The risks of hyperglycemia and hypoglycemia are obstacles to patients trying to achieve and maintain healthy blood glucose levels. Type 1 patients must be attuned to their treatment needs in order to avoid such risks, which can prove very difficult with inevitably varied lifestyles. Certain technological advances, such as the continuous glucose monitor and subcutaneous insulin infusion pumps, have aided to ease the burden of blood glucose control, but patients are still expected to intervene and ultimately are still susceptible to lapses in effective treatment. The development of the artificial pancreas (AP) is being pursued to combat this burden. Significant advancements in the development of closed-loop AP have made its use in the treatment of type 1 diabetes mellitus in real-world settings more feasible. A closed-loop AP system consists of a continuous glucose monitor, subcutaneous insulin infusion pump, and an automated control algorithm to bridge the communication gap between the two devices. To date, a number of promising AP clinical trials have taken place to test the safety and efficacy of closed-loop systems. Current studies are now beginning to transition from more inpatient to outpatient settings of larger sample sizes and longer durations, and initial studies of nonsupervised use of AP systems at home in real-life environments have already been performed. As technology and positive evidence for closed-loop therapy progresses, attitudes of acceptance toward the use of AP by patients in the real world are growing as well (1). Single-hormone, closed-loop systems are close to becoming a reality in the world of treatment for patients with type 1 diabetes mellitus, signaling AP research to transition its focus to the development of closed-loop, bihormonal systems. Our aim in this article is to present a few of the most important studies related to closed-loop AP published within the previous year.

\section{Key Articles Reviewed for the Article}

Use of an artificial pancreas among adolescents for a missed snack bolus and an underestimated meal bolus

Cherñavvsky $D R^{l}$, DeBoer $M D^{1,2}$, Keith-Hynes $P^{l}$, Mize $B^{l}$, McElwee $M^{l}$, Demartini $S^{1,2}$, Dunsmore $S F^{l}$, Wakeman $C^{l}$, Kovatchev $B P^{l}$, Breton $M D^{l}$

Pediatr Diabetes 2014. [Epub ahead of print] DOI 10.1111/pedi.12230.

\footnotetext{
${ }^{1}$ Diabetes Technology Center, Jesse Z and Sara Lea Shafer Institute for Endocrinology and Diabetes, Schneider Children's Medical Center of Israel, Petah Tikva, Israel.

${ }^{2}$ William Sansum Diabetes Center, Santa Barbara, CA.

${ }^{3}$ Department of Chemical Engineering and the Institute for Collaborative Biotechnologies, University of California at Santa Barbara, Santa Barbara, CA.

${ }^{4}$ John A. Paulson School of Engineering and Applied Sciences, Harvard University, Cambridge, MA.
} 
Inpatient trial of an artificial pancreas based on multiple model probabilistic predictive control with repeated large unannounced meals

Cameron $F^{1}$, Niemeyer $G^{2}$, Wilson $D M^{3}$, Bequette $W B^{l}$, Benassi $K S^{3}$, Clinton $P^{3}$, Buckingham $B A^{3}$

Diabetes Technol Ther 2014; 16: 728-34

A randomized trial of a home system to reduce nocturnal hypoglycemia in type 1 diabetes

Maahs DM ${ }^{I}$, Calhoun $P^{2}$, Buckingham $B A^{3}$, Chase $H P^{l}$, Hramiak $I^{4}$, Lum $\mathrm{J}^{2}$,

Cameron $F^{5}$, Bequette $B W^{5}$, Aye $T^{3}$, Paul $T^{4}$, Slover $R^{l}$, Wadwa $R P^{l}$, Wilson $D M^{3}$,

Kollman $C^{3}$, Beck $R W^{2}$

Diabetes Care 2014; 37: 1885-91

Day and night home closed-loop insulin delivery in adults with type 1 diabetes: three-center randomized crossover study

Leelarathna $L^{1,2}$, Dellweg $S^{3}$, Mader JK ${ }^{4}$, Allen $J^{1}{ }^{1}$, Benesch $C^{3}$, Doll $W^{4}$,

Ellmerer $M^{4}$, Hartnell $S^{2}$, Heinemann $L^{3}$, Kojzar $H^{4}$, Michalewski $L^{3}$, Nodale $M^{1}$,

Thabit $H^{1,2}$, Wilinska $M E^{1}$, Pieber $T R^{4}$, Arnolds $S^{3}$, Evans $M L^{1,2}$, Hovorka $R^{1}$

Diabetes Care 2014; 37: 1931-37

Overnight glucose control with an automated, unified safety system in children and adolescents with type 1 diabetes at diabetes camp

Ly $T T^{l, 2}$, Breton $M D^{3}$, Keith-Hynes $P^{3}$, De Salvo $D^{l}$, Clinton $P^{l}$, Benassi $K^{l}$, Mize $B^{3}$, Cherñavvsky $D^{3}$, Place $\mathrm{J}^{4}$, Wilson $\mathrm{DM}^{1}$, Kovatchev $B P^{3}$, Buckingham $B A^{1}$

Diabetes Care 2014; 37: 2310-16

Safety of outpatient closed-loop control: first randomized crossover trials of a wearable artificial pancreas

Kovatchev $B P^{l}$, Renard $E^{2}$, Cobelli $C^{3}$, Zisser $H^{4}$, Keith-Hynes $P^{1}$, Anderson $S^{l}{ }^{1}$ Brown $S A^{1}$, Cherñavvsky $D R^{1}$, Breton $M D^{1}$, Mize $L B^{1}$, Farret $A^{2}$, Place $J^{2}$,

Bruttomesso $D^{3}$, Del Favero $S^{3}$, Boscari $F^{3}$, Galasso $S^{3}$, Avogaro $A^{3}$, Magni $L^{5}$,

Di Palma $F^{5}$, Toffanin $C^{5}$, Messori $M^{5}$, Dassau $E^{6}$, Doyle III $F^{6}$

Diabetes Care 2014; 37: 1789-96

MD-Logic overnight control for 6 weeks of home use in patients with type 1 diabetes: randomized crossover trial

Nimri $R^{l}$, Muller $I^{l}$, Atlas $E^{l}$, Miller $S^{1}$, Fogel $A^{l}$, Bratina $N^{2}$, Kordonouri $O^{3}$, Battelino $T^{2,4}$, Danne $T^{3}$, Phillip $M^{1,5}$

Diabetes Care 2014; 37: 3025-32

Clinical results of an automated artificial pancreas using technosphere inhaled insulin to mimic first-phase insulin secretion

Zisser $H^{1,2}$, Dassau $E^{1,2,3}$, Lee $J J^{1,2}$, Harvey $R A^{1,2}$, Bevier $W^{l}$, Doyle III $F^{1,2,3}$

J Diabetes Sci Technol 2015; 9: 564-72

Adding heart rate signal to a control-to-range artificial pancreas system improves the protection against hypoglycemia during exercise in type 1 diabetes

Breton MD, Brown SA, Karvetski CH, Kollar L, Topchyan KA, Anderson SM, Kovatchev BP

Diabetes Technol Ther 2014; 16: 506-11

Comparison of dual-hormone artificial pancreas, single-hormone artificial pancreas, and conventional insulin pump therapy for glycaemic control in patients with type 1 diabetes: an open-label randomised controlled crossover trial

Haidar $A^{1,2}$, Legault $L^{1,3}$, Messier $V^{l}$, Mitre $T M^{1,4}$, Leroux $C^{l}$, Rabasa-Lhoret $R^{1,2,5,6}$

Lancet Diabetes Endocrinol 2015: 3: 17-26 


\section{Outpatient overnight glucose control with dual-hormone artificial pancreas, single-hormone artificial pancreas, or conventional insulin pump therapy in children and adolescents with type 1 diabetes: an open-label, randomised controlled trial}

Haidar $A^{1,2}$, Legault $L^{1,3}$, Matteau-Pelletier $L^{l}$, Messier $V^{l}$, Dallaire $M^{l}$, Ladouceur $M^{4}$, Rabasa-Lhoret $R^{1,2,5,6}$

Lancet Diabetes Endocrinol 2015. [Epub ahead of print]; DOI 10.1016/S2213-8587(15)00141-2

Role of glucagon-like peptide-1 analogue versus amylin as an adjuvant therapy in type 1 diabetes in a closed loop setting with ePID algorithm

Renukuntla $V S^{1}$, Ramchandani $N^{2}$, Trast $J^{2}$, Cantwell $M^{3}$, Heptulla $R A^{1}$

J Diabetes Sci Technol 2014; 8: 1011-17

Outpatient glycemic control with a bionic pancreas in type 1 diabetes

Russell $\mathrm{SJ}^{1}$, El-Khatib $\mathrm{FH}^{2}$, Sinha $\mathrm{M}^{1}$, Magyar $\mathrm{KL}^{1}$, McKeon $\mathrm{K}^{2}$, Goergen $\mathrm{LG}^{l}$, Balliro $C^{l}$, Hillard $M A^{l}$, Nathan $D M^{l}$, Damiano $E R^{2}$

N Engl J Med 2014; 371: 313-25

A novel method to detect pressure-induced sensor attenuations (PISAs) in an artificial pancreas

Baysal $N^{l}$, Cameron $F^{l}$, Buckingham $B A^{2}$, Wilson $D M^{2}$, Chase $P H^{3}$, Maahs $D M^{3}$, Bequette $W B^{I}$

J Diabetes Sci Technol 2014; 8: 1091-96

\section{CLINICAL STUDIES-SINGLE HORMONE}

\section{Use of an artificial pancreas among adolescents for a missed snack bolus and an underestimated meal bolus}

Chernavvsky $D R^{l}$, DeBoer $M D^{1,2}$, Keith-Hynes $P^{l}$, Mize $B^{l}$, McElwee $M^{1}$, Demartini $S^{1,2}$, Dunsmore $S F^{l}$, Wakeman $C^{l}$, Kovatchev $B P^{l}$, Breton $M D^{l}$

${ }^{I}$ Center for Diabetes Technology, University of Virginia, Charlottesville, VA; ${ }^{2}$ Division of Pediatric Endocrinology, Department of Pediatrics, University of Virginia, Charlottesville, VA

Pediatr Diabetes 2014. [Epub ahead of print] DOI 10.1111/ pedi.12230.

\section{Background}

The control of type 1 diabetes mellitus (T1DM) is markedly more difficult for adolescents, with this age group typically displaying higher $\mathrm{HbAlc}$ values than earlier or later age groups. Poor control is due in part to the omission of insulin for carbohydrates ingested at snacks and meals, both in the form of missing boluses entirely and underestimating carbohydrate content and thus the dosage of insulin required. The study hypothesized that AP systems would alleviate high blood glucose (BG) in adolescents in the setting of missed boluses at snacks and underestimated boluses at mealtimes and would thus offer a possible way to improve the management of T1DM for adolescents.

\section{Methods}

The randomized cross-over trial enrolled 16 adolescents with T1DM, aged 13 to 18 years old, who had been using an insulin pump for six or more months and self-reported a history of missing insulin or underbolusing. On separate days, participants received either usual care (UC) with their home insulin pump or care with an AP system (Diabetes Assistant platform, insulin pump, and continuous glucose monitor). Before admission to the study center at 08:00 hours, participants were advised to eat breakfast at or before 06:00 hours and proceed with their usual insulin intake. Upon admission all participants were connected to a remote monitoring system. One hour after admission, all participants received an unannounced snack of $30 \mathrm{~g}$ carbohydrate without an insulin bolus. Four hours later, they received an $80 \mathrm{~g}$ lunch and only $75 \%$ of the insulin dose calculated to cover the carbohydrates. Participants on the UC day (not the AP day) received their full high $\mathrm{BG}$ correction factor at lunch. Each admission lasted around 8 hours, and BG levels were monitored hourly for $>80 \mathrm{mg} / \mathrm{dL}$ and every 15 minutes for low $B G$ values.

\section{Results}

The AP system improved the primary outcome, time spent in near normoglycemia $(70-180 \mathrm{mg} / \mathrm{dL})$, both overall and at each meal (both $P<0.05$ ). The data overall and at each meal also suggested that better control did not come at the cost of increased risk for hypoglycemia, the secondary outcome. Overall glycemic control (mean BG) was improved by approximately $40 \mathrm{mg} / \mathrm{dL}$ and percent time spent over $250 \mathrm{mg} /$ dL was decreased twofold for the AP participants.

\section{Conclusions}

The study represents the first evidence of an AP system improving BG control in adolescents following insulin omission as compared to usual care with an insulin pump. 
Since the results indicate that AP improved control without inducing hypoglycemia, this has important implications regarding the potential benefits of the future application of AP systems for adolescents, a group that is particularly at risk for poor T1DM control. Although the AP provided improvements in short-term glycemic control, further testing in longer-term settings is needed.

\section{Comment}

Developing an AP algorithm efficient enough to deal with blood glucose control in adolescents has been challenging due to their tendency to maintain poorer control than other age groups. The study aimed at exploring AP efficacy in two scenarios for adolescents (missed bolus for a $30 \mathrm{~g}$ snack and underbolusing for an $80 \mathrm{~g}$ lunch). However, significance of the study was slightly reduced since both usual care and AP participants were disadvantaged with hyperglycemia after being prohibited to bolus after the unannounced snack. Permitting usual care patients to regularly bolus would have allowed for a more effective comparison to AP care. Another disadvantage was that patients remained sedentary throughout the trial, a factor that does not mirror real-world conditions. Further studies in longer-term and less-controlled settings would be helpful on the road to addressing the challenge of AP diabetes care for adolescents.

\section{Inpatient trial of an artificial pancreas based on multiple model probabilistic predictive control with repeated large unannounced meals}

Cameron $F^{1}$, Niemeyer $G^{2}$, Wilson $D M^{3}$, Bequette $W B^{l}$, Benassi $\mathrm{KS}^{3}$, Clinton $\mathrm{P}^{3}$, Buckingham $\mathrm{BA}^{3}$

${ }^{I}$ Department of Chemical and Biological Engineering, Rensselaer Polytechnic Institute, Troy, NY; ${ }^{2}$ The Walt Disney Company, Los Angeles, CA; ${ }^{3}$ Stanford University, Stanford, $C A$

\section{Diabetes Technol Ther 2014; 16: 728-34}

\section{Background}

Several closed-loop (CL) artificial pancreas studies have shown promising results for controlling blood glucose levels in people with type 1 diabetes mellitus. However, CL systems are at a disadvantage compared with insulin dosing for a meal in open-loop systems, where insulin is normally given 20 minutes before a meal due to the delayed onset of action. The objective of this CL study was to test a multiple model probabilistic predictive control (MMPPC) algorithm that can handle large, unannounced meals.

\section{Methods}

An MMPPC algorithm in a single-hormone CL artificial pancreas was assessed on two cohorts of patients. The first cohort consisted of four adult patients. After the initial cohort the algorithm was revised to improve performance, and further assessed on six additional adult patients. Each patient participated in a trial lasting 32 hours. Large meals were standardized for each participant based on weight in order to contain $0.8-1.2 \mathrm{CHO} / \mathrm{Kg}$. These meals were administered unannounced during the trial. The controller parameters only consisted of each patient's total daily insulin dose and basal rate.

\section{Results}

On a 24-hour basis, the first cohort had mean reference and CGM readings of 179 and $167 \mathrm{mg} / \mathrm{dL}$, respectively, with $53 \%$ and $62 \%$, respectively, of readings between 70 and $180 \mathrm{mg} /$ $\mathrm{dL}$ and four treatments for hypoglycemia. The second cohort had a mean reference and CGM readings of 161 and $142 \mathrm{mg} /$ $\mathrm{dL}$, respectively, with $63 \%$ and $78 \%$, respectively, of the time spent at a normal, euglycemic blood glucose and one hypoglycemic episode. The controller improvements resulted in a $43 \mathrm{mg} / \mathrm{dL}$ average reduction in the overnight glucose levels and an associated reduction in the glycemic risk, based on low and high blood glucose risk indices, from 9.2 to 6.0 $(p>0.05)$.

\section{Conclusions}

The MMPPC algorithm tested large, unannounced meals and maintained a reasonable blood glucose level for in-clinic patients. There was only one hypoglycemic event related to the controller system in the second cohort, and long-term use of the system would result in a mean glycated hemoglobin value of $7.2 \%$.

\section{Comment}

Research shows that adolescents often forget to deliver a premeal bolus, leading to spikes in blood glucose concentrations. Predictive control models, such as the one presented in this study, have the potential to be highly beneficial in younger, more active patients who may forget to administer a proper dose of insulin before a meal. Algorithms that function without the requirement of meal announcement are a significant step forward in the world of artificial pancreas and patient usability. Additionally, the researchers are moving in the right direction in trying to modify the existing algorithm, by relaxing assumptions related to the time of day for meals, exercise, and sleep. Larger outpatient studies are needed to observe statistically significant results. The algorithm is designed to anticipate future meals during daytime (between 8 am and $5 \mathrm{pm}$ ). Further research is necessary to ensure safety in the case of a skipped meal, as is common with adolescents. Based on the limited and short study, it is a bit premature to suggest that this will result with such improvement of $\mathrm{HbA1c}$. Nevertheless, future AP systems should be designed to be able to mitigate the effects of an unannounced meal and reduce hyperglycemia exposure without inducing hypoglycemia.

\section{A randomized trial of a home system to reduce nocturnal hypoglycemia in type 1 diabetes}

Maahs DM ${ }^{1}$, Calhoun $P^{2}$, Buckingham $B A^{3}$, Chase $H P^{1}$, Hramiak $I^{4}$, Lum $J^{2}$, Cameron $F^{5}$, Bequette $B W^{5}$, Aye $T^{3}$, Paul $T^{4}$, Slover $R^{1}$, Wadwa $R P^{1}$, Wilson $D M^{3}$, Kollman $C^{3}$, Beck $R W^{2}$ 
${ }^{1}$ Barbara Davis Center for Childhood Diabetes, Aurora, CO; ${ }^{2}$ Jaeb Center for Health Research, Tampa, FL; ${ }^{3}$ Stanford University, Stanford, CA; ${ }^{4}$ St. Joseph's Health Care, London, Ontario, Canada; and ${ }^{5}$ Rensselaer Polytechnic Institute, Troy, NY

Diabetes Care 2014; 37: 1885-91

This manuscript is also discussed in the article on Diabetes Technology and Therapy in the Pediatric Age Group, p. S-96.

\section{Background}

Overnight hypoglycemia is a major concern for individuals with type 1 diabetes, and if prolonged, can lead to consequences such as loss of consciousness, seizure, or even death. Since the ideal solution for those on an insulin pump would be to suspend insulin delivery before reaching hypoglycemia, hypoglycemic prediction algorithms have been developed and tested in inpatient and pilot outpatient studies. This study was designed to assess whether nocturnal hypoglycemia could be safely and effectively reduced by insulin delivery suspension at home in a larger number of individuals over a longer period of time.

\section{Methods}

Forty-five individuals with type 1 diabetes participated in a randomized, controlled trial for 42 nights. Each night participants were randomly assigned to either having the predictive low-glucose suspend system active (intervention night) or inactive (control night). The primary outcome was defined as the proportion of nights in which one or more CGM glucose values of $\leq 60 \mathrm{mg} / \mathrm{dL}$ occurred. The duration of hypoglycemic glucose levels was also followed.

\section{Results}

Results reflect data collected from 1,912 nights. Overnight hypoglycemia with at least one CGM value of $\leq 60 \mathrm{mg} / \mathrm{dL}$ occurred in 21\% (196 of 942) of intervention nights compared to $33 \%$ (322 of 970$)$ of control nights $(P<0.001)$. Median hypoglycemia area under the curve was reduced by $81 \%$ and hypoglycemia lasting over 2 hours was reduced by $74 \%$. Mean overnight glucose was $125 \mathrm{mg} / \mathrm{dL}$ for the control arm vs. $132 \mathrm{mg} / \mathrm{dL}$ for the intervention arm $(P<0.001)$. The median morning blood glucose was $144 \mathrm{mg} / \mathrm{dL}$ following intervention nights versus $129 \mathrm{mg} / \mathrm{dL}$ following control nights $(P<0.001)$. Morning ketosis occurred $<1 \%$ of the time in each arm.

\section{Conclusions}

The use of a predictive low-glucose suspend system at home can substantially reduce the frequency and duration of nocturnal hypoglycemia without increasing morning ketosis in 15-45-year-old subjects. In addition to potentially reducing the occurrence of nocturnal hypoglycemia, a low-glucose suspend system has the potential to also reduce the fear of nocturnal hypoglycemia, which can be a significant deterrent to achieving optimal blood glucose levels.

\section{Comment}

The first generation of automated insulin delivery systems aims to reduce the occurrence and severity of hypoglycemia events. These systems include the low and predicted glucose suspension features incorporated in the sensor-augmented pump therapy. The low glucose suspend is already approved and in clinical use. The described study tests the next step in automation of insulin delivery that is to suspend insulin delivery in response to predicted predefined low sensor value. The study results showed the advantage of the system in reducing overnight hypoglycemic events.

The study had an advantage in the fact that its participants were blinded. Although the duration of insulin delivery suspension was not allowed to exceed $120 \mathrm{~min}$ in a $150 \mathrm{~min}$ window or a total of $180 \mathrm{~min}$ in one night, the frequency of nightly suspensions was not reported. The primary outcome of a single glucose value of $\leq 60 \mathrm{mg} / \mathrm{dL}$ was chosen for its simplicity and based on prior studies showing a high correlation between that outcome and hypoglycemic outcomes, but the secondary outcomes, including the threefold reduction in duration of hypoglycemia, are more clinically relevant since such an event can lead to loss of consciousness, seizure, or even death. Nevertheless, the reduction in hypoglycemia was associated with significant higher mean overnight and morning glucose levels. Therefore, more studies are needed to demonstrate that the use of this technology indeed reduces the risk of hypoglycemia but without jeopardizing the overall glycemic control. This caveat may be overcome with the use of full closed-loop that may minimize both hypo- and hyperglycemia.

\section{Day and night home closed-loop insulin delivery in adults with type 1 diabetes: three-center randomized crossover study}

Leelarathna $L^{1,2}$, Dellweg $S^{3}$, Mader $J K^{4}$, Allen $J^{1}$, Benesch $C^{3}$, Doll $W^{4}$, Ellmerer $M^{4}$, Hartnell $S^{2}$, Heinemann $L^{3}$, Kojzar $H^{4}$, Michalewski $L^{3}$, Nodale $M^{1}$, Thabit $H^{1,2}$, Wilinska $M E^{1}$, Pieber $T^{4}{ }^{4}$, Arnolds $S^{3}$, Evans $M L^{1,2}$, Hovorka $R^{1}$

${ }^{I}$ Wellcome Trust-Medical Research Clinical Institute of Metabolic Science, University of Cambridge, Cambridge, UK; ${ }^{2}$ Department of Diabetes and Endocrinology, Addenbrooke's Hospital, Cambridge University Hospitals National Health Service Foundation Trust, Cambridge, UK; ${ }^{3}$ Profil Institut für Stoffwechselforschung $\mathrm{GmbH}$, Neuss, Germany; ${ }^{4}$ Division of Endocrinology and Metabolism, Department of Internal Medicine, Medical University of Graz, Graz, Austria

\section{Diabetes Care 2014; 37: 1931-37}

\section{Background}

Achieving good glycemic control while avoiding hypoglycemia is an ongoing challenge in the care of type 1 diabetes. Closed-loop insulin delivery is an emerging treatment option to address this challenge, and previous studies have revealed promising results with regards to its safety and efficacy. However, these studies have largely taken place under controlled laboratory conditions. Since the closed-loop system would be considerably more exposed to variations in meal and exercise at home, the study was undertaken to 
evaluate the performance of day and night closed-loop insulin delivery under free-living conditions at home.

\section{Methods}

Seventeen adults with type 1 diabetes on insulin pump therapy (HbA1c $7.6 \pm 0.8 \%$ and duration of diabetes $19 \pm 9$ years) participated in a three-center randomized crossover trial. At random, participants underwent two 8-day periods (1 day at the clinical research facility followed by 7 days at home) of sensor-augmented insulin pump therapy (SAP) or automated closed-loop insulin delivery. The primary outcome was determined as the time when glucose was in the target range (3.9-10.0 $\mathrm{mmol} / \mathrm{L}[70.2-180 \mathrm{mg} / \mathrm{dL}]$ ) during the home-study phase. Secondary outcomes were mean glucose, time spent below and above target glucose range, low and high blood glucose index, and insulin delivery.

\section{Results}

In the home phase, the percentage of time when glucose was in target range was significantly higher in the closed-loop compared to SAP (median 75\% [interquartile range 61-79] vs. $62 \%$ [53-70], $P=0.005)$. Mean glucose ( 8.1 vs. $8.8 \mathrm{mmol} /$ $\mathrm{L}[157.7 \mathrm{mg} / \mathrm{dL}$ vs. $146.0 \mathrm{mg} / \mathrm{dL}], P=0.027)$ and time spent above the target range $(P=0.013)$ were lower in closed-loop, while time spent below the target was comparable for both therapies $(P=0.339)$. Closed-loop increased time in target during both daytime $(P=0.017)$ and nighttime $(P=0.013)$.

\section{Conclusions}

One week of closed-loop insulin delivery at home reduces mean glucose and increases time spent in target range compared to SAP, without increasing the risk of hypoglycemia in adults with well-controlled patients. The results demonstrate the potential benefit of day and night closed-loop under unsupervised conditions.

\section{Comment}

The study is important in demonstrating the significance of the application of closed-loop AP in free-living conditions. The increase in the primary outcome, time spent in target glucose range, suggests that closed-loop AP does have potential for success in the real world under varying conditions of diet and exercise. However, further outpatient studies of longer duration and with a larger number of subjects must be conducted to move forward with the real-world application of closed-loop AP. It would also be helpful to conduct trials with populations that do not have well-controlled users. Current trials largely enroll subjects that are highly motivated to participate and thus may be somewhat primed for success. Future studies are needed to explore the strengths of closed-loop AP for populations that are less invested in managing their diabetes.

\section{Overnight glucose control with an automated, uni- fied safety system in children and adolescents with type 1 diabetes at diabetes camp}

Ly $T T^{l, 2}$, Breton $M D^{3}$, Keith-Hynes $P^{3}$, De Salvo $D^{l}$, Clinton $P^{1}$, Benassi $K^{1}$, Mize $B^{3}$, Cherñavvsky $D^{3}$, Place $\mathrm{J}^{4}$, Wilson DM ${ }^{1}$, Kovatchev $B P^{3}$, Buckingham $B A^{l}$
${ }^{1}$ Division of Pediatric Endocrinology and Diabetes, Department of Pediatrics, Stanford University School of Medicine, Stanford, CA; ${ }^{2}$ School of Paediatrics and Child Health, The University of Western Australia, Perth, Western Australia, Australia; ${ }^{3}$ Center for Diabetes Technology, University of Virginia, Charlottesville, VA; ${ }^{4}$ Department of Endocrinology, Diabetes and Nutrition, Montpellier University Hospital, Montpellier, France

\section{Diabetes Care 2014; 37: 2310-16}

This manuscript is also discussed in the article on Diabetes Technology and Therapy in the Pediatric Age Group, p. S-96.

\section{Background}

Advances in the development of automated, closed-loop systems have demonstrated improvements in the care of type 1 diabetes, including protection against hypoglycemia and decreased mean glucose levels in controlled inpatient settings. As portable automated systems have recently been developed, studies are now transitioning from controlled, inpatient settings to larger, outpatient ones. Outpatient studies on the pediatric population is of particular interest in evaluating the safety and efficacy of an overnight closed-loop system since children and adolescents face additional challenges in obtaining optimal glucose control. These challenges are especially apparent in the environment of diabetes summer camps, where glucose control is impacted by changes in altitude, exercise, and diet. The automated unified safety system (USS) was evaluated in this study for overnight closed-loop (OCL) control in children and adolescents with type 1 diabetes participating in diabetes summer camps.

\section{Methods}

The components of the USS were the Diabetes Assistant (DiAs) platform, the Dexcom G4 Platinum glucose sensor (Dexcom), and the t:slim insulin pump (Tandem Diabetes Care). The study consisted of 20 participants with type 1 diabetes (using an insulin pump for 3 months or more) who were first randomized to OCL or sensor-augmented therapy (SAP) and then crossed over every other night to the other therapy over the course of a 5- to 6-day diabetes camp. The primary outcome was defined as the percentage of time spent in the target glucose range $(70-150 \mathrm{mg} / \mathrm{dL})$ during the overnight period. Glucose data for OCL and control groups were analyzed on an intention-to-treat basis, regardless of system status, and additionally on a per-protocol basis, which allowed for the analysis of algorithm performance through the elimination of data based on sensor error.

\section{Results}

By the end of the study, 54 nights of OCL and 52 nights of SAP were observed. On an intention-to-treat basis, the median percent time in target range $(70-150 \mathrm{mg} / \mathrm{dL})$ was $62 \%$ for OCL nights compared to 55\% for SAP nights $(P=0.233)$. The median percent time in range in the per-protocol analysis was $73 \%$ for OCL nights versus $52 \%$ for SAP nights $(P=0.037)$. Mean overnight glucose values were similar, but less time was spent in the hypoglycemic range $<50,<60$, and $<70 \mathrm{mg} / \mathrm{dL}$ during OCL $(P=0.019, P=0.009$, and $P=0.023$, respectively). 


\section{Conclusions}

Although the intention-to-treat analysis did not reveal a significant difference in time spent within range between OCL and SAP, the per-protocol analysis did reveal an increased time spent in range with OCL and a significant reduction in nocturnal hypoglycemia. Results demonstrated the efficacy of the USS algorithm in improving diabetes care during overnight periods in children and adolescents participating in a diabetes camp setting. More data are required to evaluate the safety and robustness of OCL systems before allowing them to function without remote monitoring or investigator intervention.

\section{Comment}

The study represents initial steps in the transition toward full day and night closed-loop AP studies. Although recruited subjects were noted as being representative of a typical population of children, subjects were still part of a more regimental lifestyle within the diabetes camp. While the results of increased time spent within target blood glucose range and reduction in nocturnal hypoglycemia are encouraging, this would need to be duplicated in a population of children in free-living conditions in order to evaluate the safety and efficacy of closed-loop AP. The study still exemplifies good steps toward the real-world application of closed-loop AP in children with type 1 diabetes.

\section{Safety of outpatient closed-loop control: first randomized crossover trials of a wearable artificial pancreas}

Kovatchev $B P^{1}$, Renard $E^{2}$, Cobelli $C^{3}$, Zisser $\mathrm{HC}^{4}$, Keith-Hynes $P^{1}$, Anderson $S M^{1}$, Brown $S A^{1}$, Cherñavvsky $D R^{1}$, Breton $M D^{1}$, Mize $L B^{1}$, Farret $A^{2}$, Place $J^{2}$, Bruttomesso $D^{3}$, Del Favero $S^{3}$, Boscari $F^{3}$, Galasso $S^{3}$, Avogaro $A^{3}$, Magni $L^{5}$, Di Palma $F^{5}$, Toffanin $C^{5}$, Messori $M^{5}$, Dassau $E^{6}$, Doyle III $F^{6}$

${ }^{1}$ Center for Diabetes Technology and Department of Medicine, Division of Endocrinology, University of Virginia, Charlottesville, VA; ${ }^{2}$ Department of Endocrinology, Diabetes, and Nutrition, Montpellier University Hospital, INSERM Clinical Investigation Center 1001, Institute of Functional Genomics, University of Montpellier, Montpellier, France; ${ }^{3}$ Department of Information Engineering and Department of Internal Medicine, Unit of Metabolic Disease, University of Padova, Padova, Italy; ${ }^{4}$ Sansum Diabetes Research Institute, Santa Barbara, CA; ${ }^{5}$ Department of Systems Engineering, University of Pavia, Pavia, Italy; ${ }^{6}$ Department of Chemical Engineering, University of California, Santa Barbara, Santa Barbara, $C A$

Diabetes Care 2014; 37: 1789-96

\section{Background}

The goal of this study was to perform a randomized crossover trial comparing CL control versus open-loop (OL) sensor-augmented insulin pump therapy in a supervised outpatient setting. The primary objective was focused on estimating the effect size of hypoglycemia risk reduction by using a wearable artificial pancreas.

\section{Methods}

Eighteen adult patients with type 1 diabetes mellitus completed the entire protocol at three separate sites-Universities of Virginia, Padova, and Montpellier, and Sansum Diabetes Research Institute. Each patient participated in separate, 40-hour randomized crossover trials in an outpatient setting. Patients were responsible for operating a Diabetes Assistant (DiAs), which utilized an Android smartphone as a computation and communication hub for both CL and OL systems. There were no dietary restrictions, including moderate alcohol consumption, and standardized walks were performed. A remote monitoring system was used throughout the study. Aside from assessing hypoglycemia risk reduction by using a low blood glucose index (LGBI) between the two systems, secondary objectives included frequency of hypoglycemia, time within target range, and average glycemic control.

\section{Results}

The primary outcome assessing the risk of hypoglycemia (LGBI) was significantly lower on CL systems versus OL (0.64 vs. $1.12 ; p=0.003)$. CL control also results in a twofold reduction in the frequency of hypoglycemic episodes $(\mathrm{BG}<3.9 \mathrm{mmol} / \mathrm{L}$ or $70 \mathrm{mg} / \mathrm{dL}$ ) requiring carbohydrate treatment $(p=0.02)$. In terms of secondary outcomes related to glucose control, only overage blood glucose was slightly higher for CL versus OL systems $(8.96 \mathrm{mmol} / \mathrm{L}$ or $162 \mathrm{mg} / \mathrm{dL}$ and $8.45 \mathrm{mmol} / \mathrm{L}$ or $154 \mathrm{mg} / \mathrm{dL}$, respectively, $p=0.04$ ). Additionally, no serious adverse events were reported.

\section{Conclusions}

This was the first randomized, crossover trial of supervised outpatient wearable CL control versus sensor-augmented pump therapy. CL control using a smartphone (DiAs) reduced hypoglycemia and hypoglycemia treatments within patients who had no meal restrictions. There was a slight increase in average glycemia due to overemphasis on hypoglycemia safety within the control algorithm.

\section{Comment}

The applicability of this study with the use of a smartphone control device that users managed themselves is a significant step toward unrestricted use of artificial pancreas. The primary outcome in the study also revealed strong results, although further optimization is needed on the control algorithm. Specifically, algorithm modifications are required to create a balance between hypoglycemia prevention and improvement in time spent at normal glucose concentrations. For CL systems to function in unsupervised, outpatient settings there is also a need to address limitations of the system related to limited battery life, wireless communication between devices, and the implementation of cybersecurity protocols. Long-term home use of CL control will further reveal the benefits and limitations of this system. 
MD-Logic overnight control for 6 weeks of home use in patients with type 1 diabetes: randomized crossover trial

Nimri $R^{l}$, Muller $I^{1}$, Atlas $E^{l}$, Miller $S^{l}$, Fogel $A^{l}$, Bratina $N^{2}$, Kordonouri $O^{3}$, Battelino $T^{2,4}$, Danne $T^{3}$, Phillip $M^{1,5}$

${ }^{1}$ The Jesse Z. and Sara Lea Shafer Institute for Endocrinology and Diabetes, National Center for Childhood Diabetes, Schneider Children's Medical Center of Israel, Petah Tikva, Israel; ${ }^{2}$ Department of Pediatric Endocrinology, Diabetes and Metabolism, University Medical Center, University Children's Hospital, Ljubljana, Slovenia; ${ }^{3}$ Diabetes Center for Children and Adolescents, AUF DER BULT, Kinder-under Jugendkrankenhaus, Hannover, Germany; ${ }^{4}$ Faculty of Medicine, University of Ljubljana, Ljubljana, Slovenia; ${ }^{5}$ Sackler Faculty of Medicine, Tel Aviv University, Tel Aviv, Israel

Diabetes Care 2014; 37: 3025-32

This manuscript is also discussed in the article on Diabetes Technology and Therapy in the Pediatric Age Group, p. S-94.

\section{Background}

The objective of this study was to assess the effect of the MD-Logic CL artificial pancreas system on overnight glycemic control at patients' homes for an extended period of time.

\section{Methods}

Twenty-four patients with type 1 diabetes mellitus (T1DM) participated in randomized, two-part crossover study periods. Each period included 6 weeks of consecutive CL or sensoraugmented pump (SAP) therapy at home. No specific guidelines were given to participants related to physical activity or dietary restrictions (amount, type, or timing of meals). The primary outcome assessed was the amount of time spent in nocturnal hypoglycemia (blood glucose $<70 \mathrm{mg} / \mathrm{dL}$ ). Secondary outcomes included the time spent in euglycemia $(70-140 \mathrm{mg} / \mathrm{dL})$ and time spent in high or low glucose levels (above 240 or below $50 \mathrm{mg} / \mathrm{dL}$ ).

\section{Results}

Participants in the $\mathrm{CL}$ arm reduced the time spent at nocturnal hypoglycemia by a median of $40.2 \%$ when compared with individuals in the SAP arm $(p=0.02)$. The percentage of time spent in the target range also increased by a median of $21.8 \%(p=0.003)$. The average daytime glucose levels after $\mathrm{CL}$ operation were reduced by a median of $10.0 \mathrm{mg} / \mathrm{dL}$ $(p=0.017)$ and lower total daily insulin doses were observed $(p=0.038)$. No severe adverse side effects were reported in the $\mathrm{CL}$ arm.

\section{Conclusions}

This study provided a long-term analysis of the safety and efficacy of the MD-Logic CL system for individuals with T1DM during unrestricted real-life conditions. The research team demonstrated the system is effective in significantly reducing the risk of nocturnal hypoglycemia, while improving overnight glycemic control overall. Better overnight glycemic control was also seen to improve daytime control, revealing the benefit of the CL system to patients even if use is limited to nighttime periods.

\section{Comment}

Compared to other outpatient studies the 6 weeks per treatment in this study provides important information related to CL control in real-life home environments. A significant reduction in nocturnal hypoglycemia was seen, along with improved daily glycemic control with less insulin used when compared to the OL system. Better overnight control with the CL system may in turn lead to better insulin sensitivity over time for patients, although this remains to be tested. Even longer studies are needed to analyze psychosocial outcomes and quality of life related to the use of CL systems, along with an impact on HbA1c levels. Furthermore, the protocol could have been strengthened through the addition of insulin traces and variability to demonstrate the ability of the control design to manage glucose outcomes.

\section{Clinical results of an automated artificial pancreas using technosphere inhaled insulin to mimic first-phase insulin secretion}

Zisser $H^{1,2}$, Dassau $E^{1,2,3}$, Lee $J^{1,2}$, Harvey $R A^{1,2}$, Bevier $W^{l}$, Doyle III FJ $^{1,2,3}$

${ }^{1}$ Sansum Diabetes Research Institute, Santa Barbara, CA; ${ }^{2}$ Department of Chemical Engineering; ${ }^{3}$ Institute for Collaborative Biotechnologies, University of California, Santa Barbara, $C A$

\section{J Diabetes Sci Technol 2015; 9: 564-72}

\section{Background}

Unannounced meals are currently a challenge to an automated artificial pancreas without a pre-meal bolus, mainly due to the pharmacokinetics and pharmacodynamics of current available insulins. Recently, inhaled technosphere insulin (TI) has been offered as a simple and user-friendly solution to assist post-meal glycemic control. Published studies have shown that TI has a significantly shorter time to peak action when compared to regular human insulin. The goal of this clinical investigation was to assess whether adding a fixed preprandial dose of inhaled insulin to an automated CL artificial pancreas would improve postprandial glucose control without increasing the risk of hypoglycemia.

\section{Methods}

A 24-hour closed-loop study was performed on nine adult subjects with type 1 diabetes mellitus, beginning around 4:30 pm. Unannounced, mixed meals of $\sim 50$ carbohydrate (CHO) were administered at $6: 30 \mathrm{pm}$ and 7 am the following day. Additionally, patients exercised for 30 minutes at 50\% of their predicted heart rate reserve. For the treatment group, each meal was preceded by the inhalation of one $10 \mathrm{U}$ dose of TI, roughly equivalent to $4 \mathrm{U}$ of subcutaneous rapid-acting insulin. Both the control and treatment groups used a subcutaneous insulin delivery AP that was controlled by a zone model predictive algorithm. Blood glucose concentrations 
were measured every 15 minutes during hypoglycemia (BG $<70 \mathrm{mg} / \mathrm{dL}$ ) or extreme hyperglycemia (BG $>400 \mathrm{mg} / \mathrm{dL}$ ), and every 30 minutes otherwise.

\section{Results}

The use of TI increased the median percentage time in range $(70-180 \mathrm{mg} / \mathrm{dL}, \mathrm{BG})$ during the 5-hour postprandial period by $21.6 \%$ ( $81.6 \%$ and $60 \%$ for with and without TI, respectively, $p=0.06$ ). Throughout the study, median postprandial glucose was reduced from 205 to $172 \mathrm{mg} / \mathrm{dL}$ with the use of TI $(p=0.004)$. The median percentage time spent between $80-140 \mathrm{mg} / \mathrm{dL}$ over the 24 -hour period also increased by $12.3 \%$ with the use of TI $(p=0.03)$. For both the TI and the non-TI arm there was no statistical difference in the number of hypoglycemic episodes.

\section{Conclusions}

To our knowledge this was the first clinical study demonstrating that a consistent bolus of ultra-rapid, inhaled insulin to an automated AP system improves postprandial glycemic control without the need to count CHOs. The use of TI also resulted in no additional low blood glucose events or time spent in hypoglycemia.

\section{Comment}

Currently, meal control is of the most challenging obstacles related to automated insulin management systems and postprandial glycemic control. This proof of concept study demonstrates that a small dose of ultrarapid inhaled insulin improves postprandial control with a number of advantages. First, individuals using TI insulin only have to do a rough estimate of $\mathrm{CHO}$ content for every meal. Second, the use of TI is similar to first phase secretion of insulin through rapid clearance and an overall fast peak. Third, the ease of use and convenience of having an inhaled insulin option was commented on by all study participants. Future studies need to enroll a larger number of individuals and a control group is needed to compare results to conventional insulin therapy with a premeal bolus of rapid-acting insulin. In addition, researchers must determine the proportional TI dose escalation in response to larger meals and patients who have increased insulin resistance.

\section{Adding heart rate signal to a control-to-range artificial pancreas system improves the protection against hypoglycemia during exercise in type 1 diabetes}

Breton MD, Brown SA, Karvetski CH, Kollar L, Topchyan KA, Anderson SM, Kovatchev BP

Center for Diabetes Technology, University of Virginia, Charlottesville, VA

Diabetes Technol Ther 2014; 16: 506-11

\section{Background}

Preventing hypoglycemia during and after exercise is a significant challenge for people with type 1 diabetes mellitus
(T1DM). Recent closed-loop AP studies have shown decreased risks of hypoglycemia for T1DM subjects, increased amount of time spent in near-normoglycemia $(70-180 \mathrm{mg} /$ $\mathrm{dL}$ ), and reduced average glucose levels in both hospital and homelike settings, with and without exercise. This pilot feasibility study assessed whether heart rate (HR), a measurable marker for physical activity, could inform the AP algorithm and thereby improve the avoidance of hypoglycemia during and after exercise.

\section{Methods}

The study had a randomized crossover design, comparing a previously tested control-to-range (CTR) AP system with an enhanced version of the same system, including the HR signal (CTR + HR). The CTR algorithm was implemented by the DiAs portable AP platform based on the Android smartphone. Twelve adult participants with type 1 diabetes were admitted for two 26-hour periods, with each period including 30 minutes of mild exercise. Blood glucose (BG) decline during exercise was assessed, along with the Low BG Index (LBGI) (a measure of hypoglycemic risk), number of hypoglycemic episodes, and overall glucose control (percentage of time within the target range).

\section{Results}

The addition of HR to the CTR algorithm significantly reduced the average maximum $\mathrm{BG}$ decline during exercise $(P=0.022)$. LGBI was reduced marginally during exercise $(P=0.3)$, fewer hypoglycemic events were observed (none vs. two events, $P=0.16)$, and overall percentage of time spent near-normoglycemia $(70-180 \mathrm{mg} / \mathrm{dL})$ was consistently (though not significantly) higher during the CTR + HR admission ( $81 \%$ vs. $75 \%, P=0.2$ ). Overall, LGBI and average $\mathrm{BG}$ remained unchanged during recovery and overnight periods.

\section{Conclusions}

The addition of HR information to the CTR algorithm reduced $\mathrm{BG}$ decline during exercise without worsening overall glycemic control. HR-informed AP systems have the potential to decrease hypoglycemic risk during and immediately after exercise.

\section{Comment}

The study suggests that heart rate (HR) data could be added to AP systems in order to decrease risk of hypoglycemia. However, this addition would require the development of a more sophisticated algorithm since HR data can vary highly depending on circumstances unrelated to exercise. A more sophisticated algorithm would need to be developed to avoid an elevated HR in certain circumstances (such as in cases of fright or nervousness) from triggering an event. The need for additional sensors in AP systems should be questioned when the result may be increasing the risk of additional adverse events. It may be useful to conduct further studies into the possible benefits of adding HR data to AP systems since this was a pilot study that was not powered to reach statistical significance. 


\section{CLINICAL STUDIES-DUAL HORMONE}

Comparison of dual-hormone artificial pancreas, single-hormone artificial pancreas, and conventional insulin pump therapy for glycaemic control in patients with type 1 diabetes: an open-label randomised controlled crossover trial

Haidar $A^{1,2}$, Legault $L^{1,3}$, Messier $V^{1}$, Mitre $T M^{1,4}$, Leroux $C^{1}$, Rabasa-Lhoret $R^{1,2,5,6}$

${ }^{1}$ Institut de Recherches Cliniques de Montreal, QC, Canada; ${ }^{2}$ Division of Experimental Medicine, Department of Medicine, McGill University, Montreal, QC, Canada; ${ }^{3}$ Montreal Children's Hospital, McGill University Health Centre, Montreal, QC, Canada; ${ }^{4}$ Department of Mathematics and Statistics, McGill University, Montreal, QC, Canada; ${ }^{5}$ Nutrition Department, Faculty of Medicine, Université de Montreal, Montreal, QC, Canada; ${ }^{6}$ Montreal Diabetes Research Center, Montreal, QC, Canada

Lancet Diabetes Endocrinol 2015: 3: 17-26

\section{Background}

Hypoglycemia remains a major obstacle for patients with type 1 diabetes mellitus (T1DM), and recently it has been suggested the use of glucagon may be a viable treatment. The objective of this crossover trial study was to compare the effectiveness of single and dual-hormone CL AP on glycemic control and hypoglycemia prevention.

\section{Methods}

A randomized crossover trial of dual-hormone artificial pancreas, single-hormone artificial pancreas, and conventional insulin pump therapy was performed. Study participants had T1DM and were 12 years or older. Thirty participants attended an inpatient research center for three separate 24-hour study visits where meals and snacks were provided, along with a controlled exercise period. The primary outcome was the time for which blood glucose levels were in the target range $(4.0-10.0 \mathrm{mmol} / \mathrm{L}$ or $70-180 \mathrm{mg} / \mathrm{dL}$ for 2 hours postprandially and $4.0-8.0 \mathrm{mmol} / \mathrm{L}$ or 70 $140 \mathrm{mg} / \mathrm{dL}$ otherwise). Secondary outcomes include time spent outside of the target range, total insulin delivery, and the number of participants who experienced hypoglycemic episodes (glucose concentration $<3.3 \mathrm{mmol} / \mathrm{L}$ or $60 \mathrm{mg} / \mathrm{dL}$ ).

\section{Results}

When combining adult and pediatric patients, analyses reveal the mean time that blood glucose levels were spent in the target range were $62 \%$ (SD 18) and 63\% (18) during visits with single-hormone and dual-hormone artificial pancreas systems, respectively, compared with $51 \%$ (19) during the conventional treatment. There was no difference in the proportion of time spent in the target range overall and during the night between the single and dual-hormone artificial pancreas systems. Compared with the conventional therapy the singlehormone system reduced the median time spent with a blood glucose level less than $4.0 \mathrm{mmol} / \mathrm{L}$ or $70 \mathrm{mg} / \mathrm{dL}$ from $13.3 \%$ to $3.1 \%$, whereas the dual-hormone system reduced the time further to $1.5 \%$. The difference between the two artificial pancreas systems was not significant.

\section{Conclusions}

There are two main approaches to control blood glucose levels with closed-loop systems. The common one is the single-hormone delivery of insulin. The second is a dualhormonal delivery approach that adds glucagon as a counterregulatory hormone together with insulin. Each approach has pros and cons. The use of glucagon has the potential to better control hypoglycemic events. Nevertheless, adding glucagon to a closed-loop system makes the system more complex and expensive. Furthermore, this approach raises concerns about glucagon stability and effectiveness over time, with the possibility that glucagon would not be constant in counteracting the over-delivery of insulin, and thus may occasionally cause hypoglycemia. Therefore, this study aims to compare the two modalities of treatment in an attempt to answer the question whether dual-hormone control will perform better than single-hormone.

This study was the first head-to-head comparison of dualhormone artificial pancreas, single-hormone artificial pancreas, and conventional insulin pump therapy. Both AP systems significantly improved glucose control and reduced the risk related to hypoglycemia, including number of events and time spent below target range. While the dual-hormone system provides higher reduction in the time spent in the hypoglycemia than the single-hormone system, the single hormone AP may be sufficient to achieve hypoglycemia-free overnight control. The takeaway message from this important study is that no actual benefit exists in terms of glycemic control between single- and dual-hormone systems. Future studies need to analyze the effects of long-term glucagon use and patient experiences related to dual-hormone systems.

\section{Outpatient overnight glucose control with dual- hormone artificial pancreas, single-hormone artifi- cial pancreas, or conventional insulin pump therapy in children and adolescents with type 1 diabetes: an open-label, randomised controlled trial}

Haidar $A^{1,2}$, Legault $L^{1,3}$, Matteau-Pelletier $L^{1}$, Messier $V^{1}$, Dallaire $M^{1}$, Ladouceur $M^{4}$, Rabasa-Lhoret $R^{1,2,5,6}$

${ }^{I}$ Institut de Recherches Cliniques de Montreal, QC, Canada; ${ }^{2}$ Division of Experimental Medicine, Department of Medicine, McGill University, Montreal, QC, Canada; ${ }^{3}$ Montreal Children's Hospital, McGill University Health Centre, Montreal, QC, Canada; ${ }^{4}$ The Research Center of the Université de Montréal, QC, Canada; ${ }^{5}$ Nutrition Department, Faculty of Medicine, Université de Montreal, Montreal, QC, Canada; ${ }^{6}$ Montreal Diabetes Research Center, Montreal, QC, Canada

Lancet Diabetes Endocrinol 2015. [Epub ahead of print]; DOI 10.1016/S2213-8587(15)00141-2

\section{Background}

Fear of nocturnal hypoglycemia remains a major barrier in the treatment of children and adolescents with type 1 diabetes mellitus (T1DM). The objective of this crossover study was to compare the effectiveness of single and dual-hormone artificial pancreas systems on nocturnal glucose control in children and adolescents in unrestricted outpatient settings. 


\section{Methods}

A randomized, crossover trial of dual-hormone artificial pancreas, single-hormone artificial pancreas, and conventional insulin pump therapy was performed. Study participants had T1DM and were aged 9-17 years. Thirty-three participants attended a diabetes camp, participating in each of the treatment regimens for three consecutive nights with unrestricted food intake and physical activity. The primary outcome was the percentage of time in which blood glucose levels were lower than $4.0 \mathrm{mmol} / \mathrm{L}$ or $70 \mathrm{mg} / \mathrm{dL}$. Secondary outcomes included time spent with blood glucose levels within target range $(4.0-8.0 \mathrm{mmol} / \mathrm{L}$ or $70-140 \mathrm{mg} / \mathrm{dL})$, time spent outside of target range, total insulin delivery, and the number of participants who experienced hypoglycemic episodes (glucose concentration $<3.1 \mathrm{mmol} / \mathrm{L}$ or $60 \mathrm{mg} / \mathrm{dL}$ ).

\section{Results}

The time spent at glucose levels lower than $4.0 \mathrm{mmol} / \mathrm{L}$ or $70 \mathrm{mg} / \mathrm{dL}$ was median $0 \%$ during nights with the dualhormone artificial pancreas, $3.1 \%$ during nights with the single-hormone artificial pancreas, and 3.4\% during nights with conventional therapy. Dual-hormone artificial pancreas increased the time spent in the target range by $8 \%$ when compared to the single-hormone system, although this was not statistically significant. Area under the curve below $3.5 \mathrm{mmol} / \mathrm{L}$ or $63 \mathrm{mg} / \mathrm{dL}$ was not different between singleand dual-hormone therapies. Additionally, four hypoglycemic events were recorded with the single-hormone system compared to no events with the dual-hormone system.

\section{Conclusions}

The study was the first head-to-head comparison of dualhormone artificial pancreas, single-hormone artificial pancreas, and conventional insulin pump therapy in children and adolescents with T1DM in outpatient settings. Results showed that the dual-hormone artificial pancreas could improve nocturnal glycemic control in children and adolescents. However, longer and larger outpatient studies are needed to analyze important endpoints, including incidence of severe hypoglycemia, HbA1c concentrations, psychosocial outcomes, and the potential for quality-of-life improvements.

\section{Comment}

These two studies bring to light the potential benefit of the dual-hormone AP, especially for individuals with T1DM who struggle with nocturnal hypoglycemia. In addition, Gingras et al. determined a dual-hormone artificial pancreas with qualitative categorization of meal size could alleviate the burden associated with carbohydrate counting without compromising glucose control (2). In the first study, the comparison between the two modalities was at inpatient settings for day and night use. This study showed that the dual-hormone treatment further reduced hypoglycemia compared to singlehormone but only during the day and marginally during the night. In the second study conducted at a diabetes camp, only nighttime was studied. This study showed the advantage of dual-hormone over single-hormone in reducing hypoglycemia below $4.0 \mathrm{mmol} / \mathrm{L}$ or $70 \mathrm{mg} / \mathrm{dL}$ but not below $3.5 \mathrm{mmol} / \mathrm{L}$ or $63 \mathrm{mg} / \mathrm{dL}$. However, longer outpatient studies with a larger number of participants are needed to confirm whether it is justifiable to add glucagon to the artificial pancreas. A number of challenging questions remain to be answered. First, what is the cost-effectiveness between the two AP systems? Second, what are patients' perspectives on using the dual-hormone system? While the dual-hormone system has been shown to reduce hypoglycemia, it also requires an additional catheter, drug manipulation, and has an increased complexity related to usability. Third, what are the clinical side effects of extended use of glucagon in patients? Further research is needed to address these questions, as well as determining the appropriate population for each of the two artificial pancreas systems. Dual-hormone systems may be beneficial to young children, individuals with hypoglycemia unawareness, and physically active patients.

\section{Role of glucagon-like peptide-1 analogue versus amylin as an adjuvant therapy in type 1 diabetes in a closed loop setting with ePID algorithm}

Renukuntla $V S^{1}$, Ramchandani $N^{2}$, Trast $J^{2}$, Cantwell $M^{3}$, Heptulla $R A^{1}$

${ }^{I}$ Department of Pediatrics, Albert Einstein College of Medicine, Bronx, NY; ${ }^{2}$ Division of Pediatric Endocrinology and Diabetes, Montefiore Medical Center, Bronx, NY; ${ }^{3}$ Medtronic Minimed, Inc, Northridge, CA

J Diabetes Sci Technol 2014; 8: 1011-17

\section{Background}

Artificial pancreas CL systems have been shown to significantly improve nocturnal hypoglycemia. However, postprandial hyperglycemia remains a challenge. In patients with type 1 diabetes mellitus there is a metabolic failure of glucagon suppression in the postprandial period, which may be a reason for blood glucose fluctuations. The objective of this study was to determine the effectiveness of adjunctive therapy with two glucagon suppressors in the CL system in attenuating postprandial glucose levels.

\section{Methods}

Ten adult subjects with type 1 diabetes mellitus on insulin pump therapy were recruited to participate in a 27 -hour closed-loop study on three separate occasions. Subjects were placed on the insulin delivery via a CL system and in a random order received a) insulin alone (control), b) exenatide and insulin, or c) pramlintide and insulin. Meals were administered at the same time each day, with a standardized carbohydrate content of 60 grams. Medications were given prior to lunch and dinner and continuous subcutaneous blood glucose concentrations were monitored.

\section{Results}

Exanatide was found to be significantly better in lowering postprandial hyperglycemia as compared to insulin 
monotherapy $(p<0.03)$ and pramlintide use $(p>0.05)$. There was no increase in hypoglycemia with either glucagon suppressors. Nevertheless, no statistical difference was found in the average glucose concentrations for the entire study period. Insulin requirements were lower in adjunctive therapy, although not statistically significant. Exanatide was found to be significantly more effective in suppressing glucagon $(p<0.03)$.

\section{Conclusion}

This was the first clinical study investigating a comparison between the effectiveness of exanatide and pramlintide in attenuating postprandial glucose levels. In a CL system it was found that both glucagon suppressors are safe, viable options for adjunctive therapy, although exenatide had a more effective blood glucose lowering effect.

\section{Comment}

This study represents a different approach to deal with the unresolved issue of postprandial hyperglycemia. The suggested approach is to add additional treatment besides insulin in order to improve postprandial glucose control. Type 1 diabetes mellitus (T1DM) is characterized by a deficiency in insulin production and glucagon deregulation. Previous studies have shown that glucagon suppressors used in T1DM adjunctive therapy resulted in an improvement in glycemic control and decreased insulin requirements. For the first time to our knowledge, this study compared two glucagon suppressors head to head. The results of the study showed that exenatide is preferable over pramlintide in reducing postprandial glucose excursions. This has implications in choosing the kind of glucagon suppressor to use not only for closed-loop systems but for other systems as well. Nevertheless, it is questionable whether adding an injection before each meal for only marginal improvement in glucose control is worthwhile. The presented approach may complicate the closed-loop treatment and add unwanted side effects. Thus, it may be a hassle for patients to use this treatment for minimal glycemic improvement. Future research should focus on analyzing the longterm effects of adjunctive dual-hormone therapy with larger cohort. In addition, long-term studies are needed to determine dosage levels related to glucagon suppressors and whether any real health benefits exist for patients.

\section{Outpatient glycemic control with a bionic pancreas in type 1 diabetes}

Russell SJ ${ }^{1}$, El-Khatib $\mathrm{FH}^{2}$, Sinha $\mathrm{M}^{1}$, Magyar $K L^{1}$, McKeon $K^{2}$, Goergen $L G^{1}$, Balliro $C^{1}$, Hillard $M A^{l}$, Nathan $D M^{1}$, Damiano $E R^{2}$

${ }^{I}$ Diabetes Unit and Department of Medicine, Massachusetts General Hospital and Harvard Medical School, Boston, MA; ${ }^{2}$ Department of Biomedical Engineering, Boston University, Boston, $M A$

N Engl J Med 2014; 371: 313-25

\section{Background}

Achieving and maintaining normoglycemic values is a constant struggle for individuals with type 1 diabetes mellitus. The recent availability of accurate continuous glucose monitoring systems has made the development of bionic endocrine pancreatic systems feasible to improve glycemic control and reduce the burden on patients. Previous inpatient studies have shown that a single bihormonal control system can effectively regulate glycemia in adults and adolescents with type 1 diabetes mellitus. Two separate studies, for adults and for adolescents, were conducted to test the safety and efficacy of automated glycemic management systems since they have not been tested under unrestricted outpatient conditions over multiday periods.

\section{Methods}

The randomized, crossover studies had similar but distinct designs. They compared glycemic control in a wearable, bihormonal, automated, "bionic" pancreas versus glycemic control with an insulin pump in 20 adults and 32 adolescents for 5 days. The automated bionic pancreas received data from a continuous glucose monitor to control the delivery of insulin and glucagon.

\section{Results}

Mean plasma glucose level in the adults was $138 \mathrm{mg} / \mathrm{dL}$ over the 5-day bionic pancreas period and mean percentage of time with a low glucose level $(<70 \mathrm{mg} / \mathrm{dL})$ was $4.8 \%$. After one day of automatic adaptation with the bionic pancreas, the mean glucose level on continuous monitoring was lower than mean level during the control period (133 \pm 13 vs. $159 \pm 30 \mathrm{mg} / \mathrm{dL}, P<0.001)$. Percentage of time with a low glucose reading was also lower $(4.1 \%$ vs. $7.3 \%, P=0.01)$. Mean plasma glucose level in the adolescents was lower during the bionic pancreas period than during the control period $(138 \pm 18$ vs. $157 \pm 27 \mathrm{mg} / \mathrm{dL}, P=0.004)$, but the percentage of time with low plasma glucose was similar for both periods $(6.1 \%$ vs. $7.6 \%, P=0.23)$. For the adolescents the mean frequency of interventions for hypoglycemia was lower during the bionic-pancreas period than during the control period (one per 1.6 days vs. one per 0.8 days, $P<0.001)$.

\section{Conclusions}

Results of the study indicated that a wearable, automated, bihormonal bionic pancreas improved mean glycemic levels as compared with an insulin pump, with less frequent hypoglycemic episodes in both adults and adolescents with type 1 diabetes mellitus.

\section{Comment}

This first outpatient study with the dual-hormone system had encouraging results but was not without its share of adverse events. Better control was demonstrated over treatment with insulin pump alone, in contrast to most closed-loop studies that tested closed-loop systems against sensor-augmented pump therapy. Nevertheless, it is surprising that although the system includes glucagon 
as a counter-regulatory hormone, still hypoglycemia was not diminished. This may have occurred due to the close supervision of the participants for hypoglycemic events. All adverse events and technical failures, currently inevitable in the development of a bihormonal, closed-loop AP system, can be found at the supplementary appendix of the original article. Although the development of such a system is a great challenge in the world of the artificial pancreas, promising research does exist to support its improvement in the future. For example, the study noted at the time that current technology was not up to par, and there were poor glucagon formulations available for AP. However, Pohl et al. have more recently demonstrated that more stable glucagon formulations can be achieved (3). As research grows, so does the hope for a future dualhormone bionic pancreas to serve as an effective means of caring for type 1 diabetes.

\section{IN SILICO STUDIES}

\section{A novel method to detect pressure-induced sensor attenuations (PISAs) in an artificial pancreas}

Baysal $N^{1}$, Cameron $F^{1}$, Buckingham BA ${ }^{2}$, Wilson $D M^{2}$, Chase $\mathrm{PH}^{3}$, Maahs $\mathrm{DM}^{3}$, Bequette $W^{1}$

${ }^{1}$ Department of Chemical and Biological Engineering, Rensselaer Polytechnic Institute, Troy, $\mathrm{NY} ;{ }^{2}$ Division of Pediatric Endocrinology and Diabetes, Stanford University, Stanford, CA; ${ }^{3}$ Barbara Davis Center for Childhood Diabetes, Aurora, $\mathrm{CO}$

\section{J Diabetes Sci Technol 2014; 8: 1091-96}

\section{Background}

Continuous glucose monitors (CGMs) provide invaluable information regarding real-time blood glucose values. However, there is a significant need to improve glucose biosensors' response to sensor attenuation. Pressure-induced sensor attenuations (PISAs) are a common problem and often occur during periods of sleep for patients. They result in undesirable insulin attenuation or suspension of insulin delivery that may result in subsequently higher than desired morning glucose levels. The objective of this study was to introduce a novel method to detect PISAs that can be used with automated glucose control or artificial pancrease (AP).

\section{Methods}

This in silico study analyzed the effectiveness of an algorithm to detect PISAs that use real-time CGM readings without knowledge of meals, insulin doses, activity, or sensor recalibrations. The PISA detection method was tested on outpatient "in-home" data from a low glucose suspend trial with over 1,125 nights of data (108,000 CGM readings). In total, 178 sets were created using different parameters for the PISA detection algorithm to determine its functional range of performance.

\section{Results}

An expert engineer identified the PISA events through manual visual inspection of all data points $3 \%$ or 3,267 CGM readings. The algorithm successfully detected $88.34 \%$ of the PISA events with a $6.96 \%$ false positive rate. The percentage of false detections could be reduced to $1.70 \%$ by modifying the algorithm parameters.

\section{Conclusion}

This study reveals a successful PISA detection algorithm that should be applied in future overnight, clinical trials involving fully automated closed-loop AP systems. The algorithm was shown in silico to significantly reduce undesired pump shut-offs that occur due to PISAs. Subsequently, this technology may lead to improving time spent in optimal glycemic range and reduce the number of hypoglycemic events in patients with type 1 diabetes mellitus.

\section{Comment}

Automated closed-loop insulin delivery systems critically depend on the input of continuous glucose monitors (CGMs). Therefore, making sensor accuracy and reliability is a fundamental need. The suggested algorithm aims to identify the issue of incorrect sensor data due to local pressure at the sensor site that causes factitious hypoglycemia. The algorithm aim to detect PISA event is based mainly on the sudden decrease in sensor glucose levels that violate physiological rate of change limits. The algorithm performance was evaluated using a large pool of data and showed good detection rate of PISA but with a relatively high false-positive rate (that means true hypoglycemic events that will be classified as PISA and therefore will not be treated). The current algorithm uses only CGM readings, and additional information about calibrations, insulin doses, meals, exercise, and when the patient plans to sleep may decrease the number of false positives. Future prospective clinical studies are needed to test the algorithm in an overnight outpatient setting. The unresolved question is what should an automated system do in the situation of an erroneous CGM reading that is detected and whether CGMs will have a pressure sensor in the future that will provide any additional data that may allow the system to predict the effect of the local pressure on glucose values.

\section{Summary}

This year continues to bring us closer to a fully automated closed-loop artificial pancreas (AP) system. Long-term outpatient studies have begun to replace research centers and diabetes camps in the study of nocturnal hypoglycemia and the efficacy of AP. This year saw adult, adolescent, and children groups achieving improved overnight glycemic control within real-life settings including exposure to alcohol, exercise, and unannounced meals. Additionally, this year saw for the first time investigative crossover studies comparing the safety and functionality of single-hormone AP, dual-hormone AP, and sensor-augmented therapy. While both AP systems improved glucose control and reduced the risk related to hypoglycemia, further studies are needed to analyze the effects of long-term effects of glucagon use and patient experiences related to dual-hormone systems. 
The work of AP researchers this past year has also opened doors for the future of AP systems and diabetes technology. First, one study incorporated a smartphone into an AP system. As AP transitions to in-home studies, the usability of such devices will be important in management and control of diabetes mellitus. Furthermore, an in silico study revealed promising results related to improving the safety of AP algorithms, specifically in response to pressure-induced sensor attenuations. In addition, heart rate information added to the control-to-range algorithm also showed a reduction in blood glucose during exercise without an increase in hypoglycemic risk. Future studies need to continue to improve upon the safety of AP systems, while incorporating real-life patient experiences and needs in the design of such technologies. Researchers have already begun to address the importance of cybersecurity in AP trials (4).

The exploration of AP in individuals with type 2 diabetes mellitus remains another area of interest. A model describing glucose-dependent and glucose-independent insulin secretion was identified and validated by one research team, although their model now needs to be tested in clinical trials (5). The same researchers analyzed the pharmacokinetics of exogenous insulin in patients with type 2 during a closed-loop trial (6). Outpatient studies geared toward statistical significance are needed to validate their results.

While there are still issues related to algorithms, control systems of the AP and hardware, this year brings the AP closer to home use for the modern day consumer.

\section{Author Disclosure Statement}

R.N.'s institution received research grant support from Abbott Diabetes, Andromeda Biotech, Animas, BMS, Eli Lilly,
Ferring, Geffen Medical, Medtronic Diabetes, MSD Merck, Novo Nordisk, Pfizer, Sanofi, and JDRF. R.N. is a shareholder of DreaMed-Diabetes LTD. E.D. received material support from Roche Diagnostics, DexCom, Inc Animas Corp., and Lifescan Inc. E.D. is a consultant for Animas Corp. H.Y. and B.S. have no conflicts of interest.

\section{References}

1. Bevier WC, Fuller SM, Fuller RP, Rubin RR, Dassau E, Doyle FJ, Jovanovič L, Zisser HC. Artifical pancreas (AP) clinical trials' participants acceptance of future AP technology. Diabetes Technol Ther 2014; 16: 590-95.

2. Gingras V, Rabasa-Lhoret R, Messier V, Ladouceur M, Legault L, Haidar A. Efficacy of dual-hormone artificial pancreas to alleviate the carbohydrate-counting burden of type 1 diabetes: A randomized crossover trial. Diabetes Metab 2015 [Epub ahead of print]; DOI: 10.1016/j.diabet 2015.05.001.

3. Pohl R, Li M, Krasner A, De Souza E. Development of stable liquid glucagon formulations for use in artificial pancreas. J Diabetes Sci Technol 2015; 9: 8-16.

4. O'Keeffe DT, Maraka S, Basu A, Keith-Hynes P, Kudva YC. Cybersecurity in artificial pancreas experiments. Diabetes Technol Ther 2015; 17: 1-3.

5. Ruan Y, Thabit H, Wilinska ME, Hovorka R. Modelling endogenous insulin concentration in type 2 diabetes during closed-loop insulin delivery. Biomed Eng 2015; 14: 19 .

6. Ruan Y, Thabit H, Kumareswaran K, Hovorka R. Pharmacokinetics of insulin lispro in type 2 diabetes during closedloop insulin delivery. Comput Methods Programs Biomed 2014; 117: 298-307. 